\title{
ORTHOMAPS ON FORMALLY REAL SIMPLE JORDAN ALGEBRAS
}

\author{
Gregor Dolinar, Bojan KuZMa And NiK Stopar
}

Abstract. We characterize maps on finite-dimensional formally real simple Jordan algebras with the property $\phi(A \circ B)=\phi(A) \circ \phi(B)$ for all $A, B$. Although we do not assume additivity it turns out that every such map is either a real linear automorphism or a constant function. The main technique is a reduction to orthomaps, that is, maps which preserve zeros of Jordan product.

Mathematics subject classification (2010): 17C20, 17C25, $39 \mathrm{~B} 52$.

Keywords and phrases: Formally real Jordan algebra, general preserves of zeros of Jordan product, Jordan maps.

\section{REFERENCES}

[1] B. R. Bakhadly, A. E. Guterman, O. V. Markova, Graphs defined by orthogonality, J. Math. Sci. (N.Y.), 207 (2015), no. 5, 698-717.

[2] J. L. Brenner, Matrices of Quaternions, Pacific J. Math. 1, No. 3, (1951), 329-335.

[3] K. McCrimmon, A Taste of Jordan Algebras, Springer-Verlag, New York, 2004.

[4] G. Dolinar, B. Kuzma, N. STOPAR, The orthogonality relation classifies formally real simple Jordan algebras, submitted.

[5] G. Dolinar, B. KuzMa, N. Stopar, Characterization of orthomaps on the Cayley plane, Aequationes mathematicae 92 (2018), 243-265.

[6] J. Faraut, A. Korányi, Analysis on symmetric cones, Oxford Mathematical Monographs, 1994.

[7] C. A. FAURE, An elementary proof of the fundamental theorem of projective geometry, Geom. Dedicata 90, (2002), 145-151.

[8] A. Fošner, B. Kuzma, T. Kuzma, N.-S. Sze, Maps preserving matrix pairs with zero Jordan product, Linear and Multilinear Algebra 59 (2011), 507-529.

[9] F. R. Harvey, Spinors and Calibrations, Academic Press, San Diego, 1990.

[10] P. JORDAN, J. VON NEUMANN, E. WigneR, On an algebraic generalization of the quantum mechanical formalism, Ann. Math., 36 (1934), 29-64.

[11] P. JORDAN, Über Verallgemeinerungsmöglichkeiten des Formalismus der Quantenmechanik, Nachr. Ges. Wiss. Göttingen (1933), 209-217.

[12] L. Rodman, Topics in quaternion linear algebra, Princeton University Press, New-Jersey, 2014. 